\title{
Analytical and experimental investigations of dual-plane particle image velocimetry
}

\author{
Markus Raffel \\ Deutsche Forschungsanstalt für Luft- \\ und Raumfahrt \\ Institut für Strömungsmechanik \\ Center for Quantitative Visualisation \\ Bunsenstraße 10 \\ D-37073 Göttingen, Germany \\ E-mail: markus.raffel@dlr.de
}

\author{
Jerry Westerweel \\ Delft University of Technology \\ Laboratory for Aero and Hydrodynamics \\ Rotterdamseweg 145 \\ 2628 AL Delft, The Netherlands \\ Christian Willert \\ Deutsche Forschungsanstalt für Luft- \\ und Raumfahrt \\ Institut für Strömungsmechanik \\ Center for Quantitative Visualisation \\ Bunsenstraße 10 \\ D-37073 Göttingen, Germany

\section{Mory Gharib} \\ California Institute of Technology \\ Graduate Aeronautical Laboratory \\ Center for Quantitative Visualisation \\ Pasadena, California 91125 \\ Jürgen Kompenhans, MEMBER SPIE \\ Deutsche Forschungsanstalt für Luft- \\ und Raumfahrt \\ Institut für Strömungsmechanik \\ Center for Quantitative Visualisation \\ Bunsenstraße 10 \\ D-37073 Göttingen, Germany
}

\begin{abstract}
In its classical form particle image velocimetry (PIV) extracts two components of the flow velocity vector by measuring the displacement of tracer particles within a double-pulsed laser light sheet. The method described in this paper is based on the additional recording of a third exposure of the tracer particles in a parallel light sheet, which is slightly displaced with respect to the first one. The particle images resulting from these three exposures are stored on separate frames. The locations of the correlation peaks, as obtained by cross-correlation methods, are used to determine the projections of the velocity vectors onto the plane between the two light sheets. The amplitudes of these peaks are used to obtain information about the velocity component perpendicular to the light-sheet planes. The mathematical background of this method is described. Numerical simulations show the influence of the main parameters (e.g., light-sheet thickness, light-sheet displacement and out-of-plane flow component) on the resolution and reliability of the new method. A new recording procedure and its results will be shown to demonstrate the ease of operation when applying this technique to liquid flows. (c) 1996 Society of Photo-Optical Instrumentation Engineers.
\end{abstract}

Subject terms: particle image velocimetry; dual-plane correlation; scanning light sheets; three-dimensional flow measurements.

Paper 03075 received July 17, 1995; revised manuscript received Dec. 6, 1995; accepted for publication Dec. 6, 1995. This paper is a revision of a paper presented at the SPIE conference on Optical Techiques in Fluid, Thermal, and Combustion Flow, July 1995, San Diego, CA. The paper presented there appears (unrefereed) in SPIE Proceedings Vol. 2546.

\section{Introduction}

In particle image velocimetry (PIV) the velocity vector field is usually determined by subdividing the recordings into small interrogation areas and employing particle tracking algorithms or statistical evaluation techniques, such as histogram analysis, analysis of Young's fringes or other correlation methods. The correlation methods are widely used, as they can easily be implemented into fast, reliable and fully automatic evaluation systems. Their theory is well known and described by various authors. ${ }^{1-5}$ The method described in this paper is based on the spatial crosscorrelation of particle images stored on separate frames of each exposure as described by Willert and Gharib. ${ }^{6}$ It is expanded by the analysis of the heights of the peaks in the correlation plane. This value depends on the portion of paired particle images, which itself depends on the out-ofplane velocity component, but also on other parameters, e.g., background light, varying image size and number, and loss of pairs due to in-plane motion. To circumvent such problems, images from a displaced light-sheet plane, parallel to the first one, are also recorded for peak height normalization. In the remainder of this paper this concept will be referred to as dual-plane PIV. The recording of images of particles within a parallel light sheet on a third frame offers the following advantages: (1) the influence of the loss of image pairs due to the in-plane velocity on the outof-plane velocity estimation is eliminated by normalization; (2) there is no directional ambiguity with respect to the out-of-plane velocity component; (3) a larger out-of-plane velocity component can be tolerated than with conventional PIV; and (4) a higher signal-to-noise ratio for the out-ofplane velocity estimation is achieved. The mathematical concept of dual-plane PIV is described in Section 2. An optimization of the experimental parameters with respect to the image density and measurement accuracy was done by means of numerical simulations, and is described in Section 3. In contrast to earlier work ${ }^{7}$ - where specially adapted algorithms were used for the evaluation-the three image 
frames were treated as two independent PIV measurements during the first step of evaluation. The output of the evaluation of the first and second frames and of the second and third frames (i.e., the correlation peak heights and locations) were then processed with a separate computer program. The algorithm for this program is very simple, and it can easily be implemented. This procedure-together with a simplified scanning mechanism described in Sec. 4-allows easy entry into this new technique.

\section{Concept}

In this section we present the mathematical concept for the dual-plane PIV method. We follow the notation and the mathematical concepts for single-plane PIV set out by Adrian $^{1}$ and Westerweel ${ }^{5}$; for those who require a concise review of the single-plane PIV concepts we refer to the Appendix.

The dual-plane correlation technique considers three frames $i_{0}(x, y), i_{1}(x, y)$ and $i_{2}(x, y)$ that contain images of tracer particles suspended in the observed fluid. The three frames are recorded at times $t_{0}, t_{1}$ and $t_{2}$ respectively, with $t_{1}-t_{0}=t_{2}-t_{1}=\Delta t$. The particles are illuminated by light sheets that are parallel to the image plane. The intensity profiles in the direction perpendicular to the image plane are represented by $I_{0}(Z), I_{1}(Z)$ and $I_{2}(Z)$ respectively. The shape of these profiles is assumed to be identical for all three exposures; the locations of the light-sheet midplanes in the $Z$-direction for $I_{0}(Z), I_{1}(Z)$ and $I_{2}(Z)$ are $Z_{0}, Z_{1}$ and $Z_{2}$ respectively, so that

$I_{0}\left(Z-Z_{0}\right)=I_{1}\left(Z-Z_{1}\right)=I_{2}\left(Z-Z_{2}\right)=I_{Z}(Z)$.

The displacement vector $\mathbf{D}$ of the tracer particles in the fluid is considered uniform and constant over each time interval $\Delta t$. (This condition is usually satisfied locally.) Hence, the relationship between the tracer patterns (see Appendix) in the fluid for the three subsequent exposures is

$G_{1}(\mathbf{X})=G_{0}(\mathbf{X}-\mathbf{D}), \quad G_{2}(\mathbf{X})=G_{1}(\mathbf{X}-\mathbf{D})$.

Now, following the analysis for single-plane PIV (see Appendix), we obtain the following results for the image cross-correlations $R_{01}(r, s)$ and $R_{12}(r, s)$ between the frame pairs $i_{0}(x, y), i_{1}(x, y)$ and $i_{1}(x, y), i_{2}(x, y)$ respectively:

$R_{01}(r, s)=R_{00}\left(r-M D_{X}, s-M D_{Y}\right) \cdot F_{O}\left(D_{Z}-Z_{1}+Z_{0}\right)$,

$R_{12}(r, s)=R_{00}\left(r-M D_{X}, s-M D_{Y}\right) \cdot F_{O}\left(D_{Z}-Z_{2}+Z_{1}\right)$

with

$F_{O}\left(D_{Z}\right)=\int I_{Z}(Z) I_{Z}\left(Z+D_{Z}\right) \mathrm{d} Z / \int\left\{I_{Z}(Z)\right\}^{2} \mathrm{~d} Z$,

where $R_{00}(r, s)$ is the image self-correlation and $F_{O}(Z)$ the loss of correlation due to out-of-plane motion (see Appendix). Provided that $R_{00}(r, s)$ and $I_{Z}(Z)$ are known, it is in principle possible to determine $\left|D_{Z}-Z_{1}-Z_{0}\right|$ from $R_{01}(r, s)$ only. However, in a practical situation the exact shape of $R_{00}(r, s)$ is unknown, because it is influenced by local gradients of the velocity field and by the local distribution of particle-image diameters; besides, evaluation of
$\left|D_{Z}-Z_{1}-Z_{0}\right|$ leaves the sign of the out-of-plane motion undetermined. Dual-plane PIV avoids these complications by taking the ratio of $R_{01}(r, s)$ and $R_{12}(r, s)$, which yields a general expression that relates $D_{Z}$ for an arbitrary lightsheet intensity profile $I_{Z}(Z)$ and given shifts $Z_{2}-Z_{1}$ and $Z_{1}-Z_{0}$ (for brevity of notation we omit the coordinates $r$ and $s)$ :

$\frac{R_{01}}{R_{12}}=\frac{F_{O}\left(D_{Z}-Z_{1}+Z_{0}\right)}{F_{O}\left(D_{Z}-Z_{2}+Z_{1}\right)}$

In a practical situation we have to estimate the two-point covariance by a finite spatial average. It can be shown that taking the ratio of the covariance estimators also compensates for the loss of correlation due to in-plane motion, if dealing with large numbers of particle images [see Appendix, Eq. (22)].

Obviously, the ability to solve for $D_{Z}$ in Eq. (5) depends on accurate knowledge of the light-sheet profile $I_{Z}(Z)$. Most CW lasers offer a sufficient beam-pointing stability, so their intensity profiles can easily be determined to find the exact relation between the out-of-plane velocity and the cross-covariance values. When using solid-state pulsed laser systems-for example a Nd:YAG laser-a pulse-topulse beam-pointing stability of up to $20 \%$ of the beam diameter has to be taken into account. If the spatial beam profile varies in time, the intensity profile should be determined simultaneously, e.g., by using a beamsplitter and a video array.

In the remainder of this section we will deal with two particular solutions of Eq. (5), namely the solutions (1) for $Z_{0}=Z_{1}<Z_{2}$ (which is the situation that was investigated in the simulations; see Sec. 3), and (2) for $Z_{0}=Z_{2}<Z_{1}$ (which is the situation during the experiments described in Sec. 4). To keep the subsequent analysis simple we consider a light sheet with a top-hat shaped intensity profile and a width $\Delta Z$, i.e.,

$I_{Z}(Z)= \begin{cases}I_{Z}, & |Z| \leqslant \Delta Z / 2 \\ 0, & \text { elsewhere }\end{cases}$

and

$F_{O}(Z)= \begin{cases}1-|Z| / \Delta Z, & |Z| \leqslant \Delta Z \\ 0 & \text { elsewhere. }\end{cases}$

For $Z_{0}=Z_{1} \leqslant Z_{2}$, the solution of Eq. (5) for the out-ofplane velocity $w=D_{Z} / \Delta t$, with $F_{O}(Z)$ given by Eq. (7), yields

$$
w=\frac{\Delta Z}{\Delta t} \cdot \begin{cases}\frac{R_{12}-O_{Z} R_{01}}{R_{01}-R_{12}} & \text { for }-\Delta Z \cdot O_{Z} \leqslant D_{Z} \leqslant 0, \\ \frac{R_{12}-O_{Z} R_{01}}{R_{01}+R_{12}} & \text { for } 0 \leqslant D_{Z} \leqslant Z_{2}-Z_{1}, \\ \frac{R_{12}+\left(2-O_{Z}\right) R_{01}}{R_{01}-R_{12}} & \text { for } Z_{2}-Z_{1} \leqslant D_{Z} \leqslant \Delta Z\end{cases}
$$


Raffel et al.: Analytical and experimental investigations of dual-plane PIV

Table 1 Overview of the parameter sets that were simulated.

\begin{tabular}{lccccccccccc}
\hline \hline$O_{Z}$ & 0.0 & 0.2 & 0.4 & 0.6 & 0.8 & & & & & \\
$N_{l}$ & 15 & 20 & & & & & & & & \\
$D_{X} / \Delta X$ & 0.0 & 0.1 & 0.2 & 0.4 & & & & & & \\
$D_{Z} / \Delta Z$ & 0.0 & 0.1 & 0.2 & 0.3 & 0.4 & 0.5 & 0.6 & 0.7 & 0.8 & 0.9 & 1.0 \\
\hline \hline
\end{tabular}

with $O_{Z}=1-\left|Z_{2}-Z_{1}\right| / \Delta Z$. In the simulations described in Sec. 3 we will only consider the solution for $0 \leqslant D_{Z} \leqslant Z_{2}-Z_{1}$ (which is also identical to the equation originally derived by Raffel et al. ${ }^{7}$ ).

For a situation where the out-of-plane fluid velocity can be either positive or negative, a reverse displacement of the light-sheet positions between the first and second and between the second and third exposure has to be taken into account. If the absolute value of displacement is the same for both directions - as is the case for the experiment described in Sec. 4-Eq. (5) has to be solved for $Z_{0}=Z_{2}<Z_{1}$. The solution, with $F_{O}(Z)$ again given by Eq. (7), differs slightly from the solution given by Eq. (8), i.e.,

$$
\begin{aligned}
w= & \frac{\Delta Z}{\Delta t} \cdot \frac{R_{12}-R_{01}}{R_{12}+R_{01}} \cdot O_{Z} \\
& \text { for }-\left(Z_{1}-Z_{2}\right) \leqslant D_{Z} \leqslant Z_{1}-Z_{2} .
\end{aligned}
$$

Note that the range of $D_{Z}$ for this solution is symmetric with respect to $D_{Z}=0$.

\section{Simulations}

In this section we use numerical simulations to determine the reliability and accuracy of the dual-plane PIV measurements as a function of the experimental parameters. Apart from the experimental parameters that we already know from single-plane PIV, ${ }^{2,3}$ i.e. the image density $\left(N_{I}\right)$, the in-plane displacement $\left(D_{X}\right)$ with respect to the width of the interrogation region $(\Delta X)$ and the out-of-plane displacement $\left(D_{Z}\right)$ with respect to the thickness of the light sheet $(\Delta Z)$, respectively, we should now also investigate the influence of the sheet overlap $\left(O_{Z}\right)$. For this purpose we carried out a series of numerical simulations of dual-plane PIV with $Z_{0}=Z_{1}<Z_{2}$; see Eq. (8). The sets that were simulated are listed in Table 1 . The total number of parameter combinations is equal to 349; for each combination a triplet of digital image frames is generated and subsequently analyzed. For simplification the in-plane displacement was assumed to have only an $X$-component.

For each simulation a set of random particle locations is generated within a rectangular volume, where the number density of the particles matches a given image density $\left(N_{I}\right)$. Each particle is assigned a brightness value, determined by its location within the light sheet; subsequently, a digital image frame that contains 512 by 512 pixels is computed for the given particle locations and their assigned brightness values. A Gaussian intensity distribution with an $e^{-2}$ width of 1.2 pixels is assumed for each particle image; the actual pixel value is found by integration of a twodimensional Gaussian intensity distribution over the area of each pixel (integrals of a Gaussian distribution can be expressed conveniently as error functions). For the second and third images the particle locations are uniformly translated over a predefined displacement prior to computing the image frame. For the first and second images the location of the light sheet remains unchanged, whereas for the third image it is adjusted according to the value for the lightsheet overlap $\left(O_{Z}\right)$, i.e. $\left(1-O_{Z}\right) \Delta Z$. The light-sheet intensity profile is uniform within a width $\Delta Z$ and is equal to 1 ; the diameter of an interrogation area (in simulation units) is also taken equal to 1 .

For each triplet, the first and second images and the second and third images are analyzed using the crosscorrelation method. This yields two data sets that consist of the location of the displacement-correlation peak and its height for each interrogation position. Each interrogation is done over areas of 32 by 32 pixels with a $50 \%$ overlap between adjacent interrogation areas. So the total number of interrogations per image is 961 (of which only 256 are truly independent). Despite the high image density, there remains a finite probability for the occurrence of spurious displacements. To detect spurious vectors, each displacement vector is compared with the known displacement, and all vectors that deviate by more than 0.5 pixel from the true value are discarded from the data set. (Only for simulated data-where the actual displacement is known-it is possible to use such a strict selection criterion.) The remaining data from the two data sets are used to reconstruct the outof-plane component of the displacement.

\subsection{Results of Simulation}

Using the same software for the dual-plane PIV as for the single-plane PIV [i.e., with separate computation of $R_{01}(r, s)$ and $R_{12}(r, s)$, and separate identification of the correlation peaks], requires that both displacements need to be valid for reconstruction of the out-of-plane velocity component. The valid data yield, or success rate, $\Gamma$ for an image frame pair is proportional to the image density $N_{I}$, the in-plane loss of pairs $F_{I}$ and the out-of-plane loss of pairs $F_{O}$, with $F_{O}=1-\left|D_{Z}\right| / \Delta Z$ for the first and second image frames, and $F_{O}=\left(O_{Z}+\left|D_{Z}\right| / \Delta Z\right)$ for the second and third image frames. Note that for this case a small $D_{Z}$ will lead to a high valid data yield for the first and second frames, but at the same time a low valid data yield for the second and third frames. Therefore it is necessary to determine the optimum situation in which the data yield for each corresponding correlation pair is maximal. Also, it is necessary to determine for which set of parameters the measurement errors are minimal. If $\Gamma$ is plotted against the product of $N_{I} F_{I} F_{O}$, then all data practically collapse onto a single curve, ${ }^{2}$ as shown in Fig. 1. This curve corresponds to the probability curve for the presence of at least four particle images per interrogation area, given that the number of particle images has a Poisson distribution. ${ }^{5}$ 


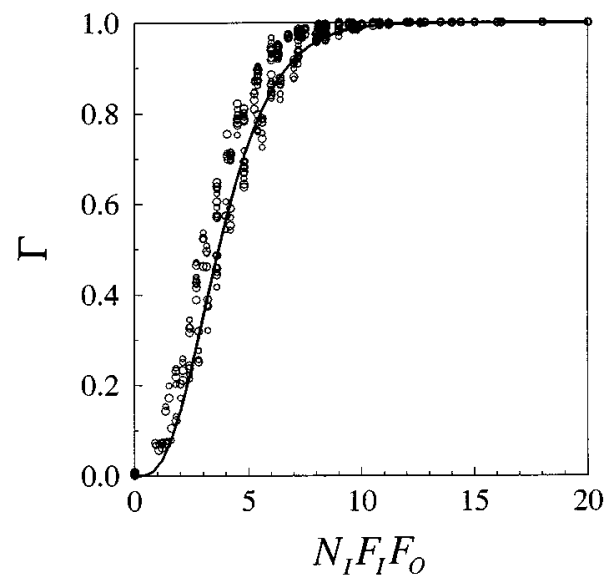

Fig. 1 The valid data yield $(\Gamma)$ as a function of the effective number of particle images $\left(N_{l} F_{l} F_{O}\right)$. The solid line represents the probability of having at least four particle images.

The fraction where both interrogations were valid is given by the product of the valid data yield for the first and second images $\left(\Gamma_{01}\right)$ and the valid data yield for the second and third images $\left(\Gamma_{12}\right)$. Figure 2 shows the valid data yield of the reconstructed displacement vectors as a function of the out-of-plane displacement $\left(D_{Z}\right)$ with respect to the center of the light-sheet overlap region [i.e., $\frac{1}{2}\left(1-O_{Z}\right) \Delta Z$ ]. This graph only shows the results from the simulations with $F_{I}=1$ (i.e. $D_{X} / \Delta X=0$ ) and $N_{I}=15$; the results for other values of $F_{I}$ and $N_{I}$ are similar. A substantial valid data yield (i.e. $\Gamma>0.75$ ) is only found for

$\left|\frac{D_{Z}}{\Delta Z}-\frac{1}{2}\left(1-O_{Z}\right)\right|<\frac{1}{4}$.

(Note that for $O_{Z}=1$ this relation reduces to the singleplane PIV optimization. ${ }^{2,3}$ )

In Fig. 3 the results for the root-mean-square (RMS)

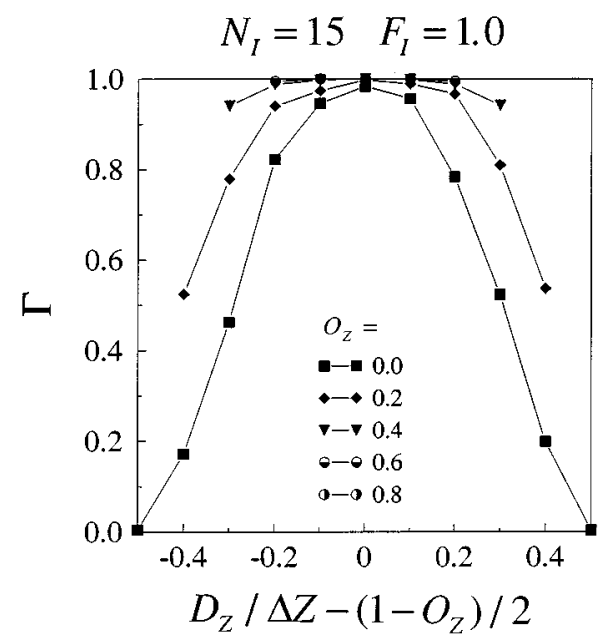

Fig. 2 The valid data yield of the reconstructed displacements $\left(\Gamma_{\text {rec }}\right)$ as a function of the out-of-plane displacement $\left(D_{Z}\right)$ with respect to the center of the overlap region of the light sheets $\left[0.5\left(1-O_{Z}\right) \Delta Z\right]$ for different values of $O_{Z}$ with $F_{l}=1$ and $N_{l}=15$.



Fig. 3 The results for the RMS error for the in-plane displacement as a function of the out-of-plane displacement for different in-plane displacement with $N_{l}=15$ and $O_{Z}=0.0$.

error for the in-plane displacement as a function of the out-of-plane displacement are plotted for different in-plane displacements with directly adjacent sheets $\left(O_{Z}=0\right)$. Graphs for other values of $O_{Z}$ show the same qualitative behavior. This graph shows that the RMS error for the measured in-plane displacement is lowest for $D_{X}=0$, which agrees with earlier results by Willert and Gharib $^{6}$ and Westerweel. ${ }^{5}$ Also note how the error becomes almost zero for $D_{Z} / \Delta Z=0.5$; this will be discussed further below.

Since we are dealing with cross-correlation analysis of pairs of single-exposure image frames, it is always possible to shift the interrogation images in the two image frames with respect to each other in such a manner that the effective particle-image displacement is equal to zero. We therefore consider only the case for $D_{X}=0$ in the remainder of the discussion of the simulation results.

For implementation of the dual-plane PIV considered in the simulations, the out-of-plane displacement should always lie between 0 and $\left(1-O_{Z}\right) / \Delta Z$; see Eq. (8). To compare the mean and RMS fluctuating values of the (reconstructed) out-of-plane displacement, each value is therefore shifted by $\frac{1}{2}\left(1-O_{Z}\right) / \Delta Z$. Figure 4 shows the difference between the observed mean and given out-of-plane displacement (i.e., $\left\langle D_{Z}\right\rangle-D_{Z}$ ) as a function of the given outof-plane displacement $\left(D_{Z}\right)$ with respect to the center of the overlap region $\left[\frac{1}{2}\left(1-O_{Z}\right) / \Delta Z\right]$ for values of $O_{Z}$ between 0.0 and 0.8 . For the range of $D_{Z}$ specified by Eq. (10) the bias error contained in the measured out-of-plane displacement is less than $2 \%$ of $\Delta Z$. Figure 5 shows the RMS error of the reconstructed out-of-plane displacement as a function of $D_{Z}$ with respect to the center of the overlap region for the same range $O_{Z}$ as in Fig. 4. This shows that the RMS error for the reconstructed out-of-plane displacement lies between $4.0 \%$ and $7.5 \%$ of $\Delta Z$ for the condition given in Eq. (10), with the smaller value corresponding to $O_{Z}=0.0$ and the higher value to $O_{Z}=0.8$. This implies that the precision can be improved by reducing $O_{Z}$ to the minimum necessary to cover the dynamic range of the out-ofplane motion without leaving the range given in Eq. (10). Note how the RMS value drops to almost zero for 


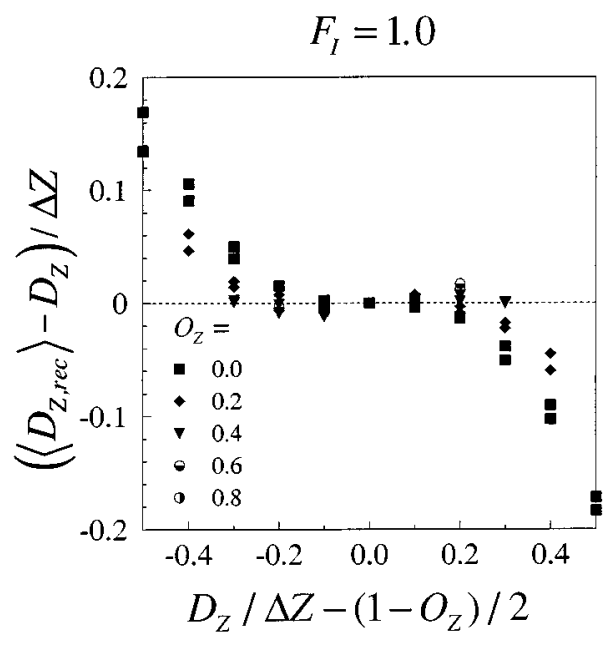

Fig. 4 The difference between the mean of the reconstructed outof-plane displacement and the given out-of-plane displacement, normalized by $\Delta Z$, as a function of the given out-of-plane displacement with respect to the center of the light-sheet overlap, normalized by $\Delta Z$, for different values of $O_{Z}$ with $F_{l}=1$.

$D_{Z}=\frac{1}{2}\left(1-O_{Z}\right) \Delta Z$. The small RMS value can be explained by the fact that the first and third images of the triplet are identical for $D_{X} / \Delta X=0$ and $D_{Z}=\frac{1}{2}\left(1-O_{Z}\right) \Delta Z$.

The results from the simulations provide insight into the optimal values for relevant experimental parameters, when using dual-frame cross-correlation analysis and combining the results of peak height and location for the first and second pair of image frames by an additional program. It was found that under the assumption of a top-hat intensity profile:

- The measurement range for the out-of-plane displacement is given by Eq. (10).

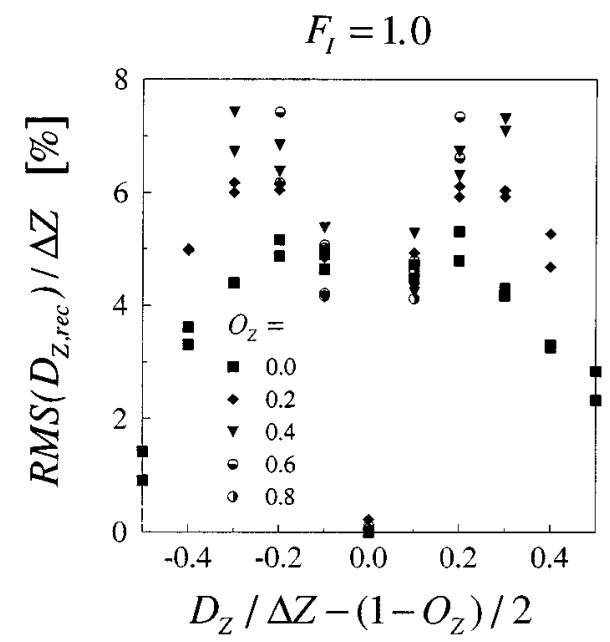

Fig. 5 The RMS error of the reconstructed out-of-plane displacement, scaled by $\Delta Z$, as a function of the given out-of-plane displacement $\left(D_{Z}\right)$ with respect to the center of the overlap region $\left[0.5\left(1-O_{Z}\right) \Delta Z\right.$ ], scaled by $\Delta Z$, for different values of $O_{Z}$ with $F_{l}=1$.

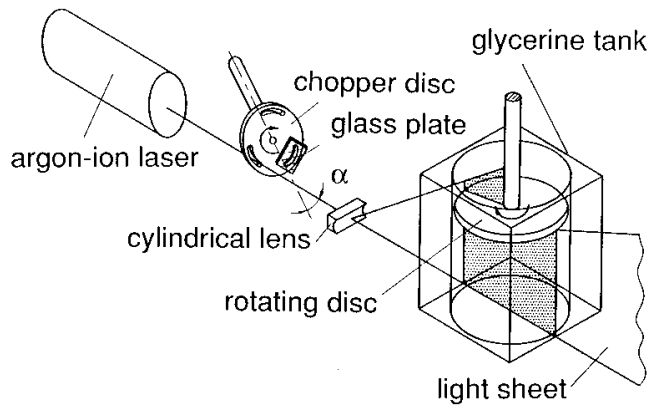

Fig. 6 Sketch of the main components of the rotating disc setup.

- For this range the bias error with respect to the given out-of-plane displacement is less than $2 \%$ of $\Delta Z$.

- The RMS value of the random error due to a finite number of particle images $\left(N_{I}=15\right)$ for the same range as given by Eq. (10) is between $4 \%$ and $8 \%$ of $\Delta Z$.

\section{Application to a Glycerine Flow Below a Rotating Disk}

In an experiment silver coated $10 \mu \mathrm{m}$ glass spheres were added to glycerine $\left(\nu=1170 \times 10^{-6} \mathrm{~m}^{2} / \mathrm{s}\right)$, which was filled into a glass tank. The flow under a rotating disc with a diameter of $170 \mathrm{~mm}$ was observed using a CCD video camera, which worked in interlaced mode and generated frames at $25 \mathrm{~Hz}$. Each frame of $752 \times 582$ pixel $^{2}$ was separated into two half-frames where the missing lines were interpolated. The stepper motor driven circular disc (angular speed: 3.1 $\mathrm{rad} / \mathrm{s}$ ) was mounted in a round glass cylinder with a diameter of $180 \mathrm{~mm}$ inside the tank, to provide circularly symmetric boundary conditions. Three-dimensional measurements by stereoscopic PIV of such a flow were previously performed by Prasad and Adrian. ${ }^{8}$

\subsection{Light-Sheet Displacement by a Chopper Disc and a Glass Plate}

The main parts of the setup are shown in Fig. 6. An argonion laser produced a continuous beam of about $1 \mathrm{~W}$ output power. A chopper disc containing three slits was driven by a stepper motor and controlled by a high precision signal generator. The rotational speed of the disc was adjusted to a light pulse frequency which was equal to the repetition rate of the video camera of 50 fields/s. It generated light pulses with a pulse length of $t_{e}=4 \mathrm{~ms}$ and a pulse separation time of $\Delta t=20 \mathrm{~ms}$. A small glass plate was mounted on the disc and covered one of the slits (see Fig. 6). This setup generated the light pulses and a parallel displacement of the light sheets.

The cylindrical lens had a focal length small enough to generate a light-sheet height approximately two times larger than the height of the observation field. The location of the light sheet alternated after each capture of a halfframe (see Fig. 7). Two subsequent full frames containing two fields each were recorded. The first and the third field contain images of tracer particles within the same light sheet oriented parallel to the axis of the disc (recorded at $t_{0}$ and $t_{2}=t_{0}+2 \Delta t$ respectively). The second field contains images of tracer particles within a light sheet slightly displaced with respect to both others (recorded at $t_{1}=t_{0}+\Delta t$ ). 


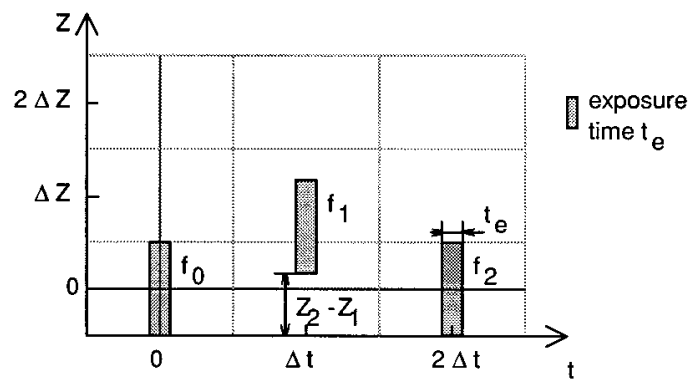

Fig. 7 Timing diagram of image capture and light-sheet position.

The shift of the light sheet was $Z_{2}-Z_{1}=0.5 \mathrm{~mm}$, resulting in an overlap of $O_{Z}=75 \%$ of the light-sheet thickness $(\Delta Z=2 \mathrm{~mm})$.

\subsection{Experimental Results on the Glycerine Flow}

Figure 8 shows the structure of the flow near the axis of rotation in a three-dimensional representation obtained by the dual-plane PIV evaluation. The out-of-plane velocity component was smoothed by a spatial averaging $(3 \times 3 \mathrm{ker}-$ nel) for this representation. The observation field had a size of $70 \times 54 \mathrm{~mm}^{2}$.

In Fig. 9 the radial $(u)$, axial $(v)$, and azimuthal $(w)$ velocity profiles of the flow under the rotating disc in a vertical distance from the disc of $5 \mathrm{~mm}$ are plotted. The solid line represents the azimuthal velocity of the disc over the radius. At a distance of $5 \mathrm{~mm}$ from the disc the radial velocity component $(u)$ varies linearly across the radius. The axial velocity component $(v)$ is small and nearly constant over the width of the observation field. The azimuthal velocity profile $(w)$ of such a flow varies linearly with decreasing angles of ascent for increasing distances from the disc (see e.g. Prasad and Adrian ${ }^{8}$ ), a trend that can also be observed in the present dual-plane PIV measurement. However, other features such as the oscillations of the azimuthal velocity profile $(w)$ cannot be explained at present

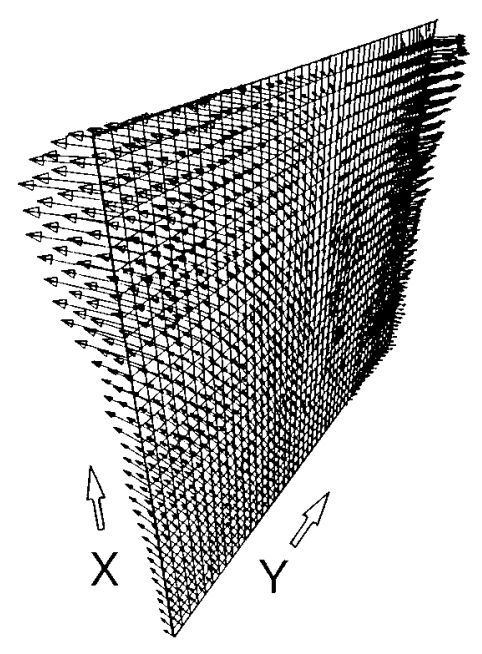

Fig. 8 Three-dimensional representation of the velocity vectors of the rotating disc flow (out-of-plane velocity component smoothed by a spatial averaging with $3 \times 3$ kernel).

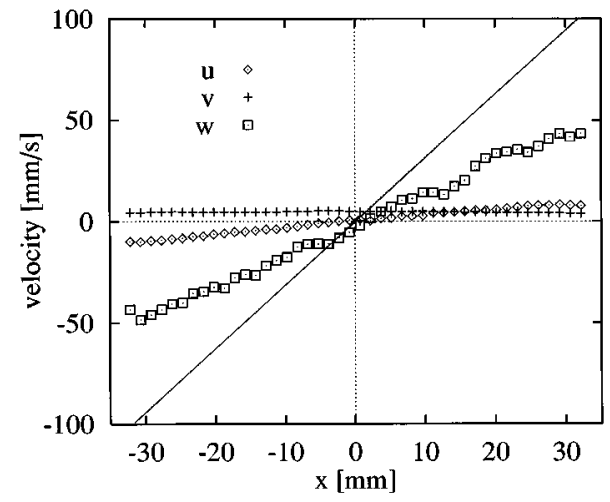

Fig. 9 Radial $(u)$, axial $(v)$ and azimuthal $(w)$ velocity profiles of the flow under the rotating disc at a vertical distance from the disc of $5 \mathrm{~mm}$ (raw data without any smoothing, data validation or interpolation).

(see Fig. 9). This may be due to imperfections of the experimental setup and to the simplification of a top-hat intensity profile of the laser beam.

\section{Conclusions}

The concept described in Sec. 2 shows that the out-of-plane velocity can be found from the ratio of the image crosscovariances (provided that the number of particle images within the interrogation area is sufficient to estimate the cross-covariances reliably). In this paper we have only presented the results for a light sheet with a top-hat shaped intensity profile. The concept presented in Sec. 2 can easily be extended to consider other light-sheet intensity profiles.

In earlier work ${ }^{7}$ the distribution containing the highest peak of each interrogated cell was used to determine the particle image displacement, and this peak position was then used to find the correct location for intensity analysis in the second cross-correlation plane. This slightly increases the dynamic range of the correlation peaks that can be evaluated, since the peak finding algorithm has to be successful only in the correlation plane with better signalto-noise ratio. However, the experimentally and numerically obtained images described in this article were treated as two independent PIV measurements during the first step of evaluation. The output of the evaluation of the first and second frame and of the second and third frame (e.g. correlation peak heights and locations) was then processed in a computer program that can easily be implemented. It was therefore no longer necessary to use completely modified evaluation software which processes three image frames at the same time.

The experimental results show the feasibility of a simple recording procedure. The results demonstrate its ease of operation when applying the technique to liquid flows. The additional displacement of the light sheet between the first two images allowed us to detect out-of-plane particle motion in both directions. When using a PIV system, which uses a CW laser in combination with a chopper disc, the only additional part needed for dual-plane PIV is a small glass plate which deflects the beam parallel. Especially when observing low speed liquid flows, the operation of dual-plane PIV is easier than that of stereoscopic PIV. The 
only calibration necessary is the determination of thickness and overlap of the light sheets. It should be emphasized that only one camera is needed.

In future investigations the influence of parameters, such as the intensity distribution of the light sheet in the $Z$ direction, and of temporal velocity gradients should be considered so as to increase the accuracy of the measurement. In contrast to stereoscopic PIV, the out-of-plane measurement error of dual-plane PIV cannot easily be estimated by analyzing the in-plane measurement error. Further work is required to improve and verify the accuracy of the technique. However, even if the accuracy of the technique presented here cannot be increased, it nevertheless reduces the number of outliers and can be used for an estimation of the out-of-plane velocity, which is adequate for many technologically important flows.

\section{Appendix}

In this appendix a concise review of the mathematical concept of single-plane PIV is given. We use the notation established by Adrian, ${ }^{1}$ but instead of the image correlation we will consider the image covariance, as set out by Westerweel. ${ }^{5}$

The single-plane correlation technique considers two frames $i_{0}(x, y)$ and $i_{1}(x, y)$ of particle images recorded at times $t_{0}$ and $t_{1}$ respectively, with $t_{1}-t_{0}=\Delta t$. The plane of the light sheet is parallel to the image plane, and the width of the light sheet is less than the focal depth of the imaging optics. The intensity of the illumination is assumed to be homogeneous with respect to the two in-plane directions; the intensity profile in the direction perpendicular to the image plane $(Z)$ is represented by $I_{Z}(Z)$. If we further assume that (1) the tracer particles all have the same diameter, that (2) the particle images do not overlap (viz., the source density $N_{S} \ll 1$ ) and (3) paraxial imaging, we can write the following expression for $i_{0}(x, y)$ :

$i_{0}(x, y)=\sum_{k} I_{Z}\left(Z_{k}\right) \cdot t\left(x-M X_{k}, y-M Y_{k}\right)$,

where $\left(X_{k}, Y_{k}, Z_{k}\right)$ is the position in the fluid of the tracer particle with index $k, M$ the image magnification, and $t(x, y)$ the normalized intensity distribution for a single particle image [and vice versa for $\left.i_{1}(x, y)\right]$. We can rewrite this expression as a convolution integral:

$i_{0}(x, y)=t(x, y) \otimes g(x, y)$

with

$g(x, y)=\frac{1}{M^{2}} \int I_{Z}(Z) G_{0}(X, Y, Z) \mathrm{d} Z$

and where $G_{0}(X, Y, Z)$ is the pattern of tracer particles in the fluid at time $t_{0}$ :

$G_{0}(X, Y, Z)=\sum_{k} \delta\left[X-X_{k}, Y-Y_{k}, Z-Z_{k}\right]$ where $\delta[X, Y, Z]$ is the Dirac $\delta$-function. For a (locally) uniform displacement field the tracer pattern that corresponds to $i_{1}(x, y)$ is equal to

$G_{1}(X, Y, Z)=G_{0}\left(X-D_{X}, Y-D_{Y}, Z-D_{Z}\right)$,

where $\left(D_{X}, D_{Y}, D_{Z}\right)$ is the (local) displacement vector of the tracer particles in the fluid.

The image cross-covariance of $i_{0}(x, y)$ and $i_{1}(x, y)$ is defined as

$R_{01}(r, s)=\left\langle i_{0}(x, y) \cdot i_{1}(x+r, y+s)\right\rangle-\left\langle i_{0}(x, y)\right\rangle\left\langle i_{1}(x, y)\right\rangle$

where $\langle\cdots\rangle$ denotes an ensemble average over all possible tracer patterns for a given flowfield. ${ }^{5}$ Substitution of $i_{0}(x, y)$ and $i_{1}(x, y)$ in (16) using the expressions given in Eqs. (12)-(15) with $R_{G_{0} G_{1}}(\mathbf{S})=C \cdot \delta(\mathbf{S}-\mathbf{D})$ yields ${ }^{5}$

$R_{01}(r, s)=R_{00}\left(r-M D_{X}, s-M D_{Y}\right) \cdot F_{0}\left(D_{Z}\right)$

with

$R_{00}(r, s)=\frac{C I_{01}^{2}}{M^{4}} t_{0}^{2} F_{t}(r, s)$,

$F_{O}\left(D_{Z}\right)=\int I_{Z}(Z) I_{Z}\left(Z+D_{Z}\right) \mathrm{d} Z / \int\left\{I_{Z}(Z)\right\}^{2} \mathrm{~d} Z$,

$F_{I}(r, s)=\iint t(u, v) t(r+u, s+v) \mathrm{d} \mu \mathrm{d} v / t_{0}^{2}$,

where $C$ is the number density of tracer particles in the fluid, $t_{0}=\iint t(x, y) \mathrm{d} x \mathrm{~d} y$, and $I_{01}^{2}=\int\left\{I_{Z}(Z)\right\}^{2} \mathrm{~d} Z$. (For the details of this derivation we refer to the theoretical analyses of Adrian ${ }^{1}$ and Westernweel. ${ }^{5}$ ) In a practical situation we have to estimate the two-point covariance by a finite spatial average over an area $A_{0}$ in $i_{0}(x, y)$ and an area $A_{1}$ in $i_{1}(x, y)$ (Refs. 1, 5):

$\hat{R}_{01}(r, s)=\iint_{A_{0}, A_{1}} i_{0}(x, y) i_{1}(x+r, y+s) \mathrm{d} x \mathrm{~d} y$.

The expectation for this estimator is equal to

$E\left\{\hat{R}_{01}(r, s)\right\}=R_{00}\left(r-M D_{X}, s-M D_{Y}\right) \cdot F_{I}(r, s) \cdot F_{O}\left(D_{Z}\right)$,

where $F_{I}(r, s)$ represents the in-plane loss of pairs. ${ }^{1,5}$ So the expectation of the estimated cross-covariance is equal to the image autocovariance shifted over the in-plane particle-image displacement, and weighted by $F_{I}(r, s)$ and $F_{O}\left(D_{Z}\right)$.

\section{Acknowledgment}

The authors would like to thank DLR for supporting Markus Raffel's visit to Caltech (CQV), and the Office of Naval Research through the URI grant ONR-URI-N0001492-J-1610. The research of J. Westerweel has been made possible by a fellowship of the Royal Netherlands Academy of Arts and Sciences. 


\section{References}

1. R. J. Adrian, "Statistical properties of particle image velocimetry measurements in turbulent flows,"' in Laser Anemometry in Fluid Mechanics III, R. J. Adrian, T. Asanuma, D. F. G. Durão, F. Durst, and J. H. Whitelaw, Eds., pp. 115-129, Springer-Verlag (1988).

2. R. D. Keane and R. J. Adrian, "Optimization of particle image velocimeters,' Meas. Sci. Technol. 1, 1202-1215 (1990)

3. R. D. Keane and R. J. Adrian, "Theory of cross-correlation analysis of PIV images,' Appl. Sci. Res. 49, 191-215 (1992).

4. K. Hinsch, "Particle image velocimetry,', in Speckle Metrology, R. S. Sirohi, Ed., Marcel Dekker, New York (1993).

5. J. Westerweel, Digital Particle Image Velocimetry. Theory and Application, Delft University Press, Delft (1993).

6. C. E. Willert and M. Gharib, "Digital particle image velocimetry," Exp. Fluids 10, 181-193 (1991).

7. M. Raffel, O. Ronneberger, M. Gharib, and J. Kompenhans, "Feasibility study of three-dimensional PIV by correlating images of particles within parallel light sheets,' Exp. Fluids 19, 69-77 (June 1995).

8. A. K. Prasad and R. J. Adrian, "Stereoscopic particle image velocimetry applied to liquid flows,' Exp. Fluids 15, 49-60 (1993).

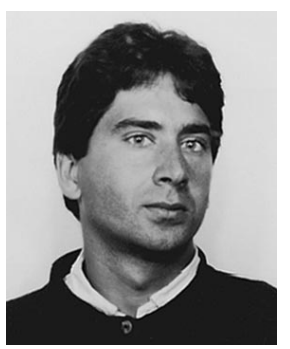

Markus Raffel received his degree in mechanical engineering from the Technical University of Karlsruhe in 1990 and his doctor's degree 1993 from the University of Hannover. He started working at DLR Göttingen in 1991 on particle image velocimetry. He is currently developing a PIV system for large wind tunnels.

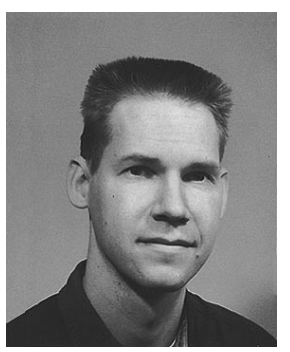

Jerry Westerweel is an assistant professor at the Laboratory for Aero and Hydrodynamics of the Delft University of Technology in the Netherlands. He received his $\mathrm{PhD}$ in mechanical engineering in 1993 and his MSc in applied physics in 1988 Both of these degrees were earned at the Delft University of Technology. His current interests include the application of particle image velocimetry to the study of turbulent flows.

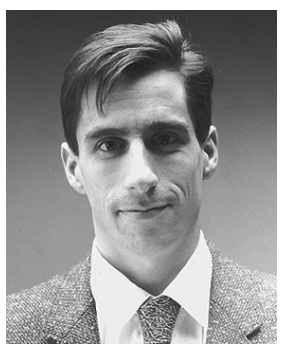

Christian Willert received his BS degree in applied science from the University of California, San Diego in 1987. Subsequent graduate work in experimental fluid dynamics (turbulence-free-surface interaction) at UCSD's Applied Mechanics and Engineering Sciences Department led to the publication of several papers on whole field measurement techniques (particle tracking velocimetry, digital PIV, 3-D particle tracking). After receiving his $\mathrm{PhD}$ in Engineering Sciences (Applied Mechanics) in 1992, he assumed postdoctoral research positions first at the Institute for Non-Linear Science (INLS) at UCSD, then at the Graduate Aeronautical Laboratories at the California Institute of Technology (Caltech). In April of 1994 he joined DLR Göttingen's measurement science group as part of an exchange program between Caltech and DLR (i.e. Center for Quantitative Visualization). There he continues to work in the development and application of PIV techniques with special emphasis on wind-tunnel applications.

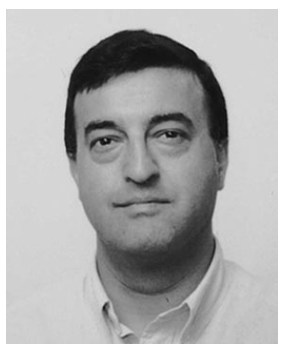

Mory Gharib is a professor of aeronautics at the California Institute of Technology. $\mathrm{He}$ received his $\mathrm{BS}$ degree in mechanical engineering from Tehran University in 1975 and then pursued his graduate studies at Syracuse University, receiving his MS in 1978 in aerospace and mechanical engineering, and his $\mathrm{PhD}$ from Caltech in 1983 in aeronautics. After two years as a senior scientist at the Jet Propulsion Laboratory (NASA/CIT), he joined the faculty of the Applied Mechanics and Engineering Sciences Department at UCSD in 1985. He became a full professor in 1992, and in January 1993 he joined Caltech as a professor of aeronautics. In 1986, he received a new-technology award from NASA for his work on the particle tracing by phosphorescent particles. His research interests have included studies of the wake of bluff bodies, advanced diagnostic systems such as multipoint laser Doppler anemometry, digital particle image velocimetry, thermometry, and 3-D particle velocimetry. Currently, he is directing the newly formed Center for Quantitative Visualization (CQV) at Caltech.

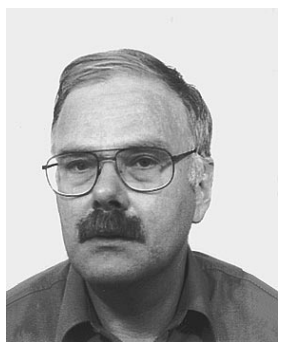

Jürgen Kompenhans received his doctor's degree in physics from the University of Göttingen in 1976. Since 1977 he has been working for Deutsche Forschungsanstalt für Luft- und Raumfahrt (DLR) as a scientist. Starting with experimental work on problems of aeroacoustics, he is now developing nonintrusive measuring techniques for aerodynamic research in wind tunnels (mainly particle image velocimetry). Presently he is working in the Institut für Strömungsmechanik at DLR, Göttingen. Since 1985 the PIV group, guided by J. Kompenhans, has developed an experimental setup for particle image velocimetry which can be operated under the rough environmental conditions of a large wind tunnel. This system has been successfully applied to low speed as well as to high speed flows (up to $500 \mathrm{~m} / \mathrm{s}$ ) in different flow facilities and for different aerodynamic applications such as boundary layer instabilities, pitching airfoils and transonic flows with shocks above airfoils. 